\title{
Aceitação de tecnologia em terminais de autosserviço aeroportuários: explorando os efeitos dos moderadores idade, experiência e gênero
}

\author{
Josivania Silva Farias ${ }^{1}$,Susy Sanders ${ }^{2}$, Carlos Denner dos Santos Jr. ${ }^{3}$, Késia \\ Rozzett $^{4}$
}

${ }^{1}$ Universidade de Brasília (UnB), Departamento de Administração (ADM), Programa de Pós-Graduação em Administração (PPGA). FACE, Caixa Postal 4320, escaninho na FACE n ${ }^{\circ}$ 72, Campus Darcy Ribeiro, Asa Norte, Brasília-DF, CEP: 70910-900.

2 Departamento de Administração (ADM), UnB

${ }^{3}$ Universidade de Brasília (UnB), Departamento de Administração (ADM), Programa de Pós-Graduação em Administração (PPGA)

${ }^{4}$ Departamento de Administração (ADM), UnB

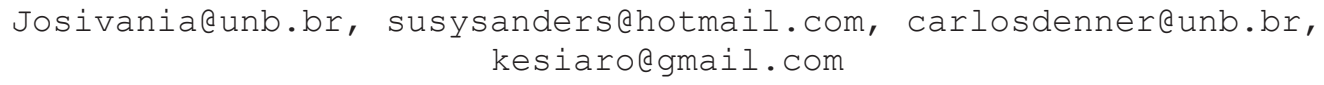

\section{RESUMO}

Exploram-se diferenças entre variáveis centrais do modelo TAM (i.e., atitude, utilidade, facilidade de uso e controle) entre passageiros agrupados conforme gênero, idade e experiência (i.e., moderadoras do modelo UTAUT). Dessa forma, promove-se uma integração original entre dois populares modelos de aceitação de tecnologia da informação. Os testes empíricos foram feitos com 456 passageiros que usaram totens de check-in em um aeroporto brasileiro, verificando-se diferenças de percepções com testes do tipo MANOVA. Facilidade de uso e controle mostram diferenças significativas, considerando todas as moderadoras; enquanto atitude e utilidade percebida não. Os resultados sinalizam: 1) a importância de se considerar as moderadoras ao mesmo tempo nos testes de hipóteses, pois algumas diferenças não são detectadas se forem estudadas isoladamente; e 2) que pesquisadores de aceitação de tecnologia devem almejar o desenvolvimento de um modelo integrador, mais genérico.

\section{ABSTRACT}

We explore the differences in key variables from TAM (i.e., attitude, usefulness, ease of use and control) between passengers grouped according to gender, age and experience (i.e., moderators from the UTAUT model). In doing that, we promote an original integration of two popular models of information technology acceptance. The empirical analysis was done with 456 passengers who used check-in totems in a Brazilian airport, verifying the differences in perceptions with MANOVA tests. Ease of use and perceived control were found to vary depending on all three moderators at once, whereas attitude and control were not. The results suggest that: 1) empirical tests must consider all moderators at once, as some differences cannot be detected if they are studied in 
isolation; and 2) that technology acceptance researchers should aim at the development of an integrative, more general, model.

\section{Introdução}

Avanços tecnológicos transformam a prestação de serviços - de encontros interpessoais para o autosserviço - o que atrai organizações, permitindo que padronizem a entrega do serviço, reduzam custos de mão-de-obra e melhorem a eficiência e a qualidade das operações, além de oferecerem novos canais de acesso aos serviços, aumentando a satisfação do cliente (Walker et al., 2002). Entretanto, em algumas ocasiões, a adoção de uma tecnologia de autosserviço não significa, necessariamente, que ela será utilizada. A relutância em adotar tecnologias, por parte de alguns consumidores, ainda é obstáculo para as empresas. Muitas tecnologias são novas, o que pode causar apreensão em consumidores que têm pouca experiência. Portanto, é recomendável analisar a atitude dos consumidores concernente a fatores que afetam sua propensão à adoção de novas tecnologias.

Várias teorias fundamentam as pesquisas sobre adoção e aceitação de TICs. Como exemplos, tem-se o Modelo de Aceitação de Tecnologias (Technology Acceptance Model), (Davis, 1986), a Teoria da Ação Racional (TRA) (Ajzen, 1975), a Teoria do Comportamento Planejado (TPB), (Ajzen, 1991) e a Unified Theory of Acceptance and Use of Technology (UTAUT) (Venkatesh, Morris, Davis e Davis, 2003), que em seguida, Venkatesh, Thong e Xu (2012) ampliaram esta teoria para o UTAUT2, cujas variáveis moderadoras do modelo foram consideradas nos testes de comparação entre percepções representadas nas médias relativas à idade, gênero e experiência de passageiros com tecnologias, como computadores.

Este estudo foi realizado em um aeroporto, próximo aos totens (terminais de autosserviço) onde usuários realizam o check-in de seus voos sem interagir com funcionários das empresas. Assim, formulou-se a seguinte questão: considerando-se gênero, idade e experiência como moderadoras da atitude, da utilidade percebida, da facilidade de uso percebida e do controle percebido em suas influências na intenção e no comportamento de uso de uma tecnologia de autosserviço, pode-se afirmar que existem diferenças de percepções entre os sujeitos a partir destas variáveis moderadoras? 
Definiu-se, então, o seguinte objetivo: verificar a existência de diferenças de percepções de usuários de tecnologias de autosserviço em aeroportos, considerando-se o gênero, a idade e a experiência dos sujeitos, como moderadoras da relação entre atitude, utilidade percebida, facilidade e controle percebidos na tecnologia analisada.

\section{Delineamento conceitual do estudo}

Tecnologias de autosserviço são "interfaces tecnológicas que permitem aos clientes produzirem um serviço sem o envolvimento direto do serviço do empregado" (Meuter et al., 2000, p.50). Exemplos incluem ATM's (automated teller machines), quiosques de informação, bancos online, totens de check-in de voos, self-service de abastecimento de combustíveis, e registros de check-out em operações no varejo (Campbell et al., 2010). Ainda assim, alguns se sentem desconfortáveis em seu uso, o que resulta em frustração em relação à tecnologia. Experimentar uma tecnologia não significa que ela será aceita, por isso, as empresas devem tornar o autosserviço atrativo, além de fácil de usar (Liljander et al., 2006).

Tecnologias de autosserviço estimulam pesquisas sobre as atitudes de consumidores em relação às mesmas. As intenções comportamentais sofrem influência das atitudes (Curran et al., 2003; Davis, 1986; Fishbein e Ajzen, 1975; Ajzen, 1991). Ao compreender as atitudes que levam às intenções de uso de tecnologias, as empresas estarão mais aptas a atingirem maior difusão dessas tecnologias.

O construto Atitude refere-se ao sentimento positivo ou negativo em relação a um comportamento (Davis, Bagozzi e Warshaw, 1989; Fishbein e Ajzen, 1975; Ajzen, 1991) e concebe as dimensões de atributos como bom-ruim, prejudicial-benéfico, agradável-desagradável, favorável-desfavorável. O componente afetivo da atitude é o sentimento da pessoa em relação a um objeto por refletir posicionamentos: "eu gosto", "eu não gosto", "eu espero" etc. Dabholkar e Bagozzi (2002) mostraram que as percepções de facilidade de uso, execução e diversão têm efeitos positivos e diretos na atitude diante de touch-screen.

As bases para a criação do modelo TAM (Modelo de Aceitação da Tecnologia (Davis, 1986) foram a Teoria da Ação Racional (TRA) (Ajzen, 1975) e a Teoria do Comportamento Planejado (Ajzen, 1991), pressupondo que indivíduos normalmente 
agem de maneira racional e fazem uso de informações disponíveis. Um comportamento depende da intenção de adotá-lo, o que é função da atitude em relação ao comportamento pretendido (Davis, 1986). A intenção de uso de uma tecnologia seria determinada pela atitude do indivíduo em relação à mesma. Essa atitude é determinada pela utilidade percebida e pela facilidade de uso percebida (Davis e Venkatesh, 1996). A utilidade percebida refere-se ao grau em que o usuário acredita que usar um sistema pode melhorar a execução de seu trabalho, e a facilidade de uso percebida refere-se ao grau em que o usuário acredita que o uso de determinado sistema amenizará seu esforço (Davis, 1986). O controle percebido se refere às crenças individuais a respeito da presença ou ausência dos recursos e oportunidades necessários à realização do comportamento (Ajzen, 1991; Lu, Chou e Ling, 2009). Dabholkar, Bobbitt e Lee (2003, p.87) estudaram fatores que influenciavam os consumidores a usar (ou evitar) check-outs de autoleitura (self-scanning check-outs) em supermercados, concluindo que a percepção de controle, a confiança, a facilidade de uso e o prazer eram importantes para os consumidores no uso dessa tecnologia baseada em autosserviço.

Venkatesh et al. (2003), desenvolveram o modelo Unified Theory of Acceptance and Use of Technology (UTAUT) a partir de revisões de oito diferentes teorias do campo de estudos de aceitação de tecnologias e comportamento humano. Assim, propuseram que: a intenção e o comportamento de uso de tecnologias são influenciados diretamente pela expectativa de performance, expectativa de esforço, influencia social e condições facilitadoras, sendo, todavia, estas relações moderadas pelo gênero, idade, experiência dos sujeitos e, em algumas situações, pela voluntariedade de uso da tecnologia. Posteriormente, Venkatesh, Thong e Xu (2012) ampliaram o modelo (UTAUT), propondo que: a intenção comportamental e o comportamento de uso de uma tecnologia são influenciadas diretamente pela expectativa de performance, expectativa de esforço, influencia social, condições facilitadoras, motivação hedônica, custo-benefício e hábito, sendo, todavia, estas relações moderadas pelo gênero, idade, experiência dos sujeitos.

Considerando-se este o arcabouço teórico, foram delineadas as seguintes hipóteses para esta pesquisa: 
$\mathrm{H}_{1}=$ Se gênero, idade e experiência de usuários de determinada tecnologia moderam a atitude como variável influenciadora de intenção e do uso, então há diferenças de percepções entre os sujeitos, considerando-se a variável influenciadora e as moderadoras citadas.

$\mathrm{H}_{2}=\mathrm{Se}$ gênero, idade e experiência de usuários de determinada tecnologia moderam a facilidade de uso percebida como variável influenciadora de intenção e do uso, então há diferenças de percepções entre os sujeitos, considerando-se a variável influenciadora e as moderadoras citadas.

$\mathrm{H}_{3}=$ Se gênero, idade e experiência de usuários de determinada tecnologia moderam a utilidade percebida como variável influenciadora de intenção e do uso, então há diferenças de percepções entre os sujeitos, considerando-se a variável influenciadora e as moderadoras citadas.

$\mathrm{H}_{4}=\mathrm{Se}$ gênero, idade e experiência de usuários de determinada tecnologia moderam o controle percebido como variável influenciadora de intenção e do uso, então há diferenças de percepções entre os sujeitos, considerando-se a variável influenciadora e as moderadoras citadas.

\section{Método}

Esta pesquisa estudou a diferença de percepções entre passageiros de diferentes grupos, considerando-se gênero, idade e experiência com aparatos tecnológicos, que são variáveis consideradas moderadoras (Venkatesh, Thong e Xu, 2012) da relação entre atitude, utilidade percebida, facilidade percebida e controle percebido (Davis, 1986; Ajzen, 1991) e a intenção e comportamento de uso de uma tecnologia de autosserviço. Para tanto, foi realizada uma pesquisa exploratório-descritiva de abordagem quantitativa de corte transversal, por meio de um levantamento (survey) com passageiros transeuntes no aeroporto internacional de Brasília-DF. Segundo Anuário Estatístico da INFRAERO, em 2009, os aeroportos brasileiros movimentaram mais de 110 milhões de passageiros. Em 2011, o movimento correspondeu a 115 milhões de passageiros. No ano 2012 registrou-se movimento correspondente a 193,8 milhões de passageiros e entre janeiro e novembro de 2013, a movimentação, com crescimento exponencial, correspondeu a 726,7 milhões de passageiros nacionais e internacionais nos aeroportos brasileiros 
(INFRAERO, 2013). No ano 2011, o Aeroporto Internacional Juscelino Kubitscheck, em Brasília, recebeu 1.277.502 de passageiros. A amostra da pesquisa foi do tipo não probabilística, por acessibilidade, visto que a coleta dependeu da disposição das pessoas em participar. A população restringiu-se a indivíduos que utilizaram pelo menos uma vez os totens de autosserviço. Da aplicação, retornaram 506 questionários respondidos, porém, 456 foram considerados válidos $(\mathrm{N}=456)$. A quantidade de missings, por questão, oscilou entre três e cinco casos omissos por questão.

O instrumento de coleta de dados foi um questionário autoaplicável, com 16 itens distribuídos entre quatro construtos estudados utilizando escala Likert, com sete níveis de respostas, desde discordo totalmente (1) até concordo totalmente (7), além de variáveis sociodemográficas e uma questão a respeito do grau de domínio no uso do computador. Este questionário foi submetido à análise de juízes com professores da Universidade de Brasília e validação semântica, por meio de pré-teste com sujeitos semelhantes aos que participariam da amostra definitiva, sendo aplicado no aeroporto citado, no período de janeiro a novembro de 2011. A análise dos dados foi feita a partir de testes de diferenças multivariadas de variância entre médias (MANOVA). Isso porque o design do estudo possuía 4 variáveis para verificação de diferenças (atitude, facilidade, controle e utilidade). Como essas variáveis eram latentes, ou seja, foram observadas através de mais de uma indicador no questionário, realizou-se uma análise fatorial com cada uma para se obter o primeiro fator, viabilizando a criação de uma nova e única variável resultante da combinação linear de todos os seus indicadores. Os testes multivariados foram seguidos de testes univariados, considerando-se cada uma das variáveis em foco isoladamente, e tornando assim necessário o controle poshoc (Bonferroni) do erro tipo I devido à grande quantidade de testes realizados. Os resultados dessas análises seguem.

\section{Resultados e Discussão}

\subsection{O perfil dos sujeitos da pesquisa}

Os sujeitos da pesquisa tinham o seguinte perfil sociodemográfico: $66 \%$ $(\mathrm{n}=301)$ eram homens e $34 \%(\mathrm{n}=155)$ mulheres; 52\% $(\mathrm{n}=237)$ tinham de 18 a 40 anos; 
$45 \%(n=205)$ tinham 41 a 63 anos e 3\% (14) tinham entre 64 e 83 anos; quanto à renda dos sujeitos da pesquisa, pode-se afirmar que era elevada, pois apenas 32\% tinham renda inferior a $\mathrm{R} \$ 4.000,00.46 \%$ afirmaram ter renda entre $\mathrm{R} \$ 4.001,00$ e $\mathrm{R} \$$ $10.000,00$ e 22\% possuíam renda acima de $\mathrm{R} \$ 10.001,00$. Quase a metade dos entrevistados (48\%) viajava de avião apenas uma vez por ano. Os demais (52\%) viajavam semanalmente, mensalmente, ou até mais de uma vez por mês. Quanto à instrução, $82 \%$ dos sujeitos já possuíam o superior completo ou incompleto e até mesmo pós-graduação. Somente $18 \%$ tinham ensino médio ou fundamental.

Para a análise dos resultados, organizou-se a base de dados por grupos para se proceder aos testes de comparação das médias de percepção dos grupos existentes: Faixa etária (3 grupos): Grupo 1: 18-40 anos; Grupo 2: 41-63 anos; Grupo 3: 64-83 anos. Gênero (2 grupos): Grupo 1- Feminino; Grupo 2- Masculino. Experiência - para esta questão, o sujeito se deparava com a seguinte pergunta no questionário: assinale o seu grau de domínio no uso de equipamentos eletrônicos, como por exemplo, o computador: (1) Não domino... 23456 ...(7) Domino. Assim, para o tratamento e análise dos dados, convencionou-se a Experiência (3 grupos) da seguinte maneira: Grupo 1 (assinalou 2 a 3): Pouca experiência; Grupo 2 (assinalou 4 a 5): Razoável experiência; Grupo 3 (assinalou 6 a 7): Muita experiência. Esta organização pode ser vista na Tabela 1 , a seguir.

Tabela 1 Distribuição de frequência dos diferentes grupos:

\begin{tabular}{|c|c|c|}
\hline GÊENERO & $N=456$ & Frequência Relativa \\
\hline Grupo 1 - Feminino & 155 & $34 \%$ \\
\hline Grupo 2 - Masculino & 301 & $66 \%$ \\
\hline$F A I X A E T A ́ R I A$ & $N=456$ & Frequência Relativa \\
\hline Grupo 1: 18-40 anos & 237 & $52 \%$ \\
\hline Grupo 2: 41-63 anos & 205 & $45 \%$ \\
\hline Grupo 3: 64-83 anos & 14 & $3 \%$ \\
\hline EXPERIÊENCIA & $N=456$ & Frequência Relativa \\
\hline Grupo 1: Pouca Experiência & 19 & $4 \%$ \\
\hline Grupo 2: Razoável Experiência & 142 & $31 \%$ \\
\hline Grupo 3: Muita Experiência & 295 & $65 \%$ \\
\hline
\end{tabular}

Considerando que o instrumento continha 3 a 4 perguntas para compor cada uma das variáveis (atitude, facilidade de uso, utilidade e controle percebidos), procedeuse à análise fatorial, a fim de extrair o componente principal de cada uma das variáveis 
latentes citadas, conforme relatado no Método. Com isto, verificou-se que a primeira assertiva ("Utilizar o totem de auto check-in em aeroportos é uma boa ideia") correspondia a $88 \%$ da variância total explicada, dispensando-se assim os demais itens relacionados à Atitude; a primeira assertiva ("Eu acho o totem de auto check-in útil") correspondia a $91,48 \%$ da variância total explicada da variável Utilidade; a primeira assertiva ("Eu acho fácil utilizar o totem de auto check-in") correspondia a 93\% da variância total explicada da variável Facilidade de Uso; e a primeira assertiva ("Eu sou confiante de que posso utilizar o totem de auto check-in corretamente") totalizava $84,2 \%$ da variância total explicada da variável Controle Percebido.

\subsection{Comparações entre as médias dos grupos}

Comparando-se as médias a partir do gênero em relação às quatro variáveis influenciadoras da intenção e uso de tecnologia de autosserviço em aeroporto, verificouse que para qualquer que seja a variável (Atitude, Facilidade de Uso, Utilidade e Controle Percebidos), quando o teste é feito com gênero, há diferenças significativas entre as médias $(\mathrm{p}<.05)$. Quanto ao teste de comparação entre médias das quatro variáveis influenciadoras da intenção e uso de tecnologia de autosserviço em aeroporto, verificou-se que para qualquer que seja a variável (Atitude, Facilidade de Uso, Utilidade e Controle Percebidos), quando o teste é feito com os três grupos de faixas etárias (Grupo 1: 18-40 anos; Grupo 2: 41-63 anos; Grupo 3; 64-83 anos;), há diferenças significativas entre as médias $(\mathrm{p}<.05)$. Já com relação ao teste de comparação entre médias das quatro variáveis influenciadoras da intenção e uso de tecnologia de autosserviço em aeroporto, verificou-se que para qualquer que seja a variável (Atitude, Facilidade de Uso, Utilidade e Controle Percebidos), quando o teste é feito com os três grupos divididos por nível de experiência (Grupo 1: assinalou 2 a 3 - Pouca experiência; Grupo 2 assinalou 4 a 5 - Razoável experiência; Grupo 3: assinalou 6 a 7 - Muita experiência), há diferenças significativas entre as médias $(\mathrm{p}<.05)$.

Oque se verifica até aqui é que quando apenas uma característica do grupo é destacada (apenas gênero, apenas idade ou apenas experiência), a comparação entre médias com quaisquer que sejam as variáveis resulta significativa $(\mathrm{p}<.05)$. Assim, foram necessários testes cruzados de comparação entre médias, considerando mais de 
uma característica de grupo, por exemplo: idade e gênero, juntos, comparando-se as médias relativas a cada uma das quatro variáveis influenciadoras da intenção e uso de tecnologia (Atitude, Facilidade de Uso, Utilidade e Controle Percebidos) e assim por diante.

O primeiro teste cruzado foi realizado com os níveis 1,2 e 3 de faixas etárias, considerando-se as quatro variáveis (Atitude, Facilidade de Uso, Utilidade e Controle Percebidos), verificando-se que apenas a variável Utilidade Percebida não apresenta diferença significativa entre médias, quando cruzadas na comparação as faixas 2 vs. 1 (41-63 anos vs. 18-40 anos), $\mathrm{p}>.05$ (Sig. $=0,100)$. Isso mostra que a hipótese $\mathrm{H}_{3}$ deste estudo não pode ser suportada, quando considerada a variável moderadora Idade, entre as faixas 2 e 1. Já para as demais variáveis (Atitude, Facilidade de Uso e Controle Percebidos), há diferenças significativas entre médias considerando-se essas duas faixas etárias. Quando se procedeu à comparação entre as faixas etárias 3 e 1, verificou-se que há diferenças significativas entre as médias $(\mathrm{p}<.05)$ em relação à percepção de cada umas das quatro variáveis influenciadoras da intenção e uso da tecnologia e isso é compreensível, uma vez que a diferença de idade entre os grupos 1 e 3 também é expressiva (faixa etária 1, de 18 a 40 anos; faixa etária 3, de 64 a 83 anos) - um grupo bem mais jovem e outro idoso.

O segundo teste cruzado foi realizado com os níveis de experiência 1 (Pouca experiência) e 2 (Razoável experiência), considerando-se as quatro variáveis (Atitude, Facilidade de Uso, Utilidade e Controle Percebidos), concluindo-se que há diferenças significativas entre sujeitos com pouca e com razoável experiência no lidar com computador $(\mathrm{p}<.05 ;$ Sig. $=.000)$. Porém, quando a comparação foi feita entre os grupos 3 (muita experiência) e 1 (Pouca experiência), os testes não resultaram significativos ( $p>.05)$, o que faz com que todas as hipóteses do estudo sejam apenas parcialmente suportadas. Isto merece atenção, pois o esperado é que a experiência (pouca ou muita) promova diferenças nas percepções dos usuários. Talvez isso possa ser explicado pelo fato de que a faixa etária e o gênero tenham que ser, sempre, considerados de modo agrupado com a variável experiência. Venkatesh, Thong e Xu (2012) hipotetizaram que homens mais velhos tendem a possuir níveis mais elevados de experiência com tecnologia. Assim, todas as hipóteses desta pesquisa podem ser suportadas, desde que a variável moderadora Experiência seja sempre considerada de modo vinculado à idade e 
ao gênero. Isoladamente, o teste sinaliza que os pouco e os muito experientes, quando colocados em comparação, não apresentam percepções distintas em relação às variáveis influenciadoras da intenção e uso de tecnologia de autosserviço em aeroporto, o que parece dissonante.

Analisando os resultados dos testes de efeito entre grupos, verificou-se que quando se vincularam as três variáveis moderadoras (gênero/faixa etária/experiência) na análise das quatro variáveis influenciadoras da intenção e uso de tecnologia, surgiram os seguintes resultados, conforme Tabela 2, a seguir:

Tabela 2 - Testes de efeitos entre grupos

\begin{tabular}{l|l|l}
\hline \multicolumn{1}{c|}{ Variáveis moderadoras } & $\begin{array}{c}\text { Variáveis influenciadoras de } \\
\text { intenção e uso de tecnologias }\end{array}$ & $\begin{array}{c}\text { Significância dos efeitos entre } \\
\text { grupos }\end{array}$ \\
\hline Gênero/Experiência/Faixa Etária & Atitude & $\mathrm{p}>.05(\mathrm{sig} .=.238)$ \\
\hline Gênero/Experiência/Faixa Etária & Utilidade & $\mathrm{p}>.05($ sig. $=.405)$ \\
\hline Gênero/Experiência/Faixa Etária & Facilidade de uso & $\mathrm{p} \leq .05($ sig. $=.057)$ \\
\hline Gênero/Experiência/Faixa Etária & Controle Percebido & $\mathrm{P}<.05($ sig. $=.002)$ \\
\hline
\end{tabular}

Assim, as variáveis: Facilidade de Uso e Controle Percebido apresentaram efeitos significativos nos testes de efeitos entre grupos. Entretanto, Atitude e Utilidade Percebida não apresentaram significância nos testes (embora quando cada uma das moderadoras seja tratada separadamente, passe a apresentar significância nos efeitos resultantes dos testes com cada uma das vaiáveis influenciadoras da intenção e uso da tecnologia estudada). Observa-se, assim, que a análise necessita ser mais complexa, envolvendo todas as variáveis moderadoras escolhidas para este trabalho.

São resultados intrigantes, uma vez que nos testes iniciais, vários resultados mostravam diferenças entre médias de percepção das variáveis influenciadoras de intenção e uso da tecnologia estudada - o que inclusive permitia concluir que era preciso trabalhar com todo o conjunto das três variáveis moderadoras. Entretanto, quando se procederam aos testes vinculando as três variáveis moderadoras comparando as percepções a partir de cada uma das variáveis influenciadoras, os testes com duas influenciadoras se mostraram significativos (Facilidade de Uso e Controle Percebido) e os testes com outras duas (Atitude e Utilidade Percebida) não foram significativos (a um IC de 95\%). Isso possibilita a não rejeição das hipóteses $\mathrm{H}_{2}$ e $\mathrm{H}_{4}$ e a rejeição das hipóteses $\mathrm{H}_{1}$ e $\mathrm{H}_{3}$ deste estudo. 


\section{Conclusões}

Esta pesquisa testou a junção de modelos de aceitação de tecnologias associados a teorias comportamentais no contexto do autosserviço em operações de check-in em aeroporto; além disso, relacionou variáveis relativas ao perfil demográfico dos participantes às suas atitudes e percepções em relação aos totens de autosserviço.

Concluiu-se que se considerando facilidade de uso e controle percebido, os testes de comparação entre médias com todo o conjunto de variáveis moderadoras (gênero, faixa etária e experiência), mostram diferenças significativas. De forma antagônica, quando consideradas as variáveis atitude e utilidade percebida, os testes sinalizam que não há diferenças entre as médias dos grupos. Entre os principais achados da pesquisa, ressalta-se a importância de estudos futuros considerarem as moderadoras em conjunto - ao mesmo tempo - nos seus testes de hipóteses, pois algumas diferenças não podem ser detectadas se as moderadoras forem estudadas isoladamente. Adicionalmente, tornou-se evidente que variáveis centrais dos modelos de aceitação de tecnologia da informação, anteriormente estudadas de forma independente, necessitam de tratamento concomitante, sinalizando a necessidade de desenvolvimento de modelo integrador, mais genérico.

Este trabalho contribui para os estudos sobre aceitação de tecnologias alinhada às variáveis atitude e controle percebido. A atitude positiva em relação ao totem de autosserviço poderá contribuir para maior adoção desta tecnologia. Por esta razão, incluiu-se o construto atitude no teste proposto.

\section{Referências}

Ajzen, I. (1991) "The Theory of planned Behavior, Organizational Behavior and Human Decision Processes”, v. 50, n. 2, p. 179-211.

Bitner, M. J.; Ostrom A. L.; Meuter, M. L. (2002) "Implementing Successful SelfService Technologies". Academy of Management Executive. v. 16, n.4, p. 96-109.

Bitner, M.J.; Brown, S.W.; Meuter, M.L. (2000) "Technology infusion in service encounters". Journal of the Academy of Marketing Science. v. 28, n.1, p.138-149.

Campbell, C.S.; Maglio, P.P.; Davis, M.M. (2010) "From self-service to super-service: a resource mapping framework for co-creating value by shifting the boundary between provider and customer". Information Systems E-business Management. P. 174-19.

Curran, J.M.; Meuter, M.L.; Surprenant, C.F. (2003) "Intentions to Use Self-Service Technologies: A Confluence of Multiple Attitudes". Journal of Service Research. v.5, n.3, p. 209-224. 
Dabholkar, P.A.; Bagozzi, R.P. (2002) "An attitudinal model of technology-based selfservice: moderating effects of consumer traits and situational factors". Journal of the Academy of Marketing Science. v.30, n.3, p. 184-201.

Dabholkar, P.A.; Bobbitt, L.M.; Lee, E.J. (2003) "Understanding consumer motivation and behavior related to self-scanning in retailing: Implications for strategy and research on technology-based self-service". International Journal of Service Industry Management. v. 14, n.1, p. 59-95.

Davis, F. D.; Venkatesh, V. (1996) "A critical assessment of potential measurement biases in the technology acceptance model: three experiments". International Journal of Human-Computer Studies. v. 145, p. 19-45.

Davis, F.D. (1986) "A technology Accepatance Model for Empirically Testing New End-User Information Systems: Theory and Results".1985. Dissertação (Doutorado em Gestão) - Sloan School of Management, Massachusetts Institute of Technology, Cambridge.

Empresa Brasileira de Infraestrutura Aeroportuária (INFRAERO) (2013) Anuário Estatístico Operacional - dez. 2013. Site da Infraero. Disponível em: < http://www.infraero.gov.br/index.php/br/estatistica-dos-aeroportos.html > . Acesso em 26 de dezembro de 2013.

Fishbein, M.; Ajzen, I. (1975) "Belief, attitude, intention, and behavior: An introduction to theory and research". Reading, MA: Addison-Wesley.

Liljander, V.; Gillberg, F.; Gummerus, J.; Riel, A. (2006) “Technology readiness and adoption of self-service technologies". Journal of Retailing and Consumer Services. v. 13, p. 177-191.

Lu, J-L.; Chou, H-Y; Ling, P-C. (2009) "Investigating passenger's intentions to use technology-based self check-in services". Transportation Research Part E. p. 345-356.

Meuter, M.L.; Ostrom, A.; Roundtree, R.; Bitner, M.J. (2000) "Self-Service Technologies: Understanding Customer Satisfaction with Technology-Based Service Encounters". Journal of Marketing, v. 64, p.50-64, jul.

Meuter, M.L; Bitner, M.J.; Ostrom, A.L.; Brown, S. (2005) "Choosing among alternative service delivery modes: an investigation of customer trial of self-service technologies". Journal of Marketing, v.69, p.61-83.

Venkatesh, V.; Morris, M. G.; Davis, G.B.; Davis, F.D.: (2003) "User Acceptance Of Information Technology: Toward A Unified View”, MIS Quarterly Vol. 27 No. 3, pp. 425-478/September.

Venkatesh, V.; Thong, J. Y. L.; Xu, X.: (2012) "Consumer Acceptance And Use Of Information Technology: Extending The Unified Theory Of Acceptance And Use Of Technology”. Forthcoming in MIS Quarterly, Vol. 36, No. 1 (2012), pp. 157-178.

Walker, R. H.; Lees M. C.; Hecker, R.; Francis, H. (2002) "Technology-Enabled Service Delivery: An Investigation of Reasons Affecting Customer Adoption and Rejection". International Journal of Service Industry Management. v. 13, n. 1, p. 91106. 